\title{
El proceso de desarrollo en las experiencias de construcción socialista de Viet Nam y Cuba: un análisis comparado
}

The development process in the socialist construction experiences of Viet Nam and Cuba: a comparative analysis

\author{
Carla Elizabeth Rodríguez Piñeiro. ${ }^{1} \&$ An Phuong Dang Do Loc. ${ }^{2}$ \\ Recibido: 02-03-2019 / Revisado: 10-03-2019 / Aceptado: 26-03-2019 / Publicado: 05-04-2019
}

Resumen.

DOI: https://doi.org/10.33262/concienciadigital.v2i2.943

En este trabajo se analiza el proceso de desarrollo en el contexto del socialismo histórico. En este sentido se exponen las experiencias vietnamita y cubana, destacando los resultados, tanto positivos como negativos de las estrategias de desarrollo adoptadas en estos países, lo cual ha guardado estrecho vínculo con las condiciones de desarrollo que prevalecen en cada región. Para ello, se empleará un modelo econométrico a partir de la herramienta del paquete informático Eviews, para medir el impacto del crecimiento obtenido en el proceso de construcción socialista sobre los niveles de desarrollo alcanzado por cada país, en términos de desarrollo humano.

Palabras claves: hegemonimo desarrollo, crecimiento, socialismo, Producto Interno Bruto (PIB) per cápita, Índice de Desarrollo Humano (IDH).

\section{Abstract.}

This paper analyses the development process of two experience of socialist construction, Viet Nam and Cuba. Also, the results, positives and negatives, of the implementation of the development strategies applied by each country, are exposed. The latest has been closely linked to the development conditions prevailing in each region. An econometric model was applied using the Eviews software package, to

\footnotetext{
${ }^{1}$ Universidad de La Habana, La Habana, Cuba, cerodriguez@ gmail.com.

${ }^{2}$ Universidad de La Habana, La Habana, Cuba, apdang@gmail.com.
} 
measure the impact of the growth obtained by each country on the development levels achieved by them, in terms of human development.

Keywords: development, growth, socialism, Per capita Gross Domestic Product (GDP), Human Development Index.

\section{Introducción.}

El socialismo y el desarrollo son dos conceptos diferentes, pero también se puede apreciar la existencia de un fuerte vínculo entre ambos. El desarrollo se caracteriza por una condición social en la cual los bienes y servicios se encuentran al alcance, de manera sostenida, de todos los individuos que integran la sociedad, lo cual conlleva a una mayor integración económica y social, implicando una disminución de las condiciones de marginalidad de determinados individuos dentro de la sociedad. Lo anterior está en concordancia con la implementación del sistema socialista, debido a que plantea una organización equitativa de la economía y una justa repartición de los bienes producidos.

La historia de los países socialista abarca algo más de cien años de la humanidad (desde 1917 hasta la actualidad). Tanto en el proceso de evolución de estos como en su período de tránsito, han mostrado determinadas complejidades que han dificultado y han hecho más lento ese largo proceso de transición hacia una sociedad socialista. Un gran debate ha surgido después de la caída del Campo Socialista a finales de los años ochenta del siglo pasado sobre cuál debería ser el camino a seguir para alcanzar el desarrollo en los marcos de construcción socialista. Por lo tanto, en la actualidad es importante estudiar experiencias de países que se encuentran en el proceso de construcción socialista y que aspiran a obtener mayores niveles de desarrollo. En este sentido se torna imprescindible tomar en consideración las diferentes condiciones, contextos históricos y los factores determinantes que responden a cada cual para seguir a un camino correcto y construir un modelo que verdaderamente genere desarrollo.

El presente trabajo tiene como objetivo analizar el proceso de desarrollo de las experiencias de construcción socialista de Cuba y Viet Nam. Por lo tanto propone en las siguientes páginas, abordar la importancia del sistema socialista para alcanzar el desarrollo; analizar la existencia de diferentes condiciones en el período de tránsito hacia el sistema socialista desde diversas regiones -con características diferentes- que han optado por ese tipo de sociedad, y su impacto en el proceso de desarrollo, explicar el impacto del crecimiento obtenido como resultado de la aplicación de políticas dirigidas a la creación de una nueva sociedad, en el desarrollo alcanzado por las experiencias vietnamita y cubana en particular.

Para ello, se efectuará una comparación de los niveles de desarrollo alcanzados por ambas experiencias. En este sentido se explora el impacto sobre los niveles de desarrollo del crecimiento obtenido por cada nación en su camino al Socialismo, mediante un análisis econométrico -utilizando como herramienta el paquete Eviews- cuyas variables 
representativas serán el Índice de Desarrollo Humano (IDH) y el Producto Interno Bruto (PIB) per cápita expresados en logaritmos.

\section{Una mirada a las experiencias de construcción de socialismo en Cuba y Vietnam. Generalidades.}

El Socialismo es una corriente política, económica y social que tiene como principales autores a Carlos Marx y Federico Engels, que desarrollaron su teoría en el auge del sistema capitalista durante el siglo XIX. Surge en oposición a la lógica del capital, como reacción a las desigualdades sociales que ha generado, fundamentalmente después de la Revolución Industrial, de modo que propone la reducción de la propiedad privada y la eliminación de las clases sociales en contraposición al Capitalismo, que se basa en la propiedad privada sobre los medios de producción y el libre comercio; considerándose, por tanto, una formación económico-social superior al sistema capitalista mundial. Tiene como base la colocación de los medios de producción al patrimonio colectivo y la administración de estos por parte del propio pueblo.

Para analizar el desarrollo de las diferentes experiencias del Socialismo histórico resulta necesario tener en cuenta que el cambio de estructura económica, la diversificación productiva y el incremento de la complejidad técnica son los factores que lo promueven; mientras que las condiciones que se requieren en cada región para propiciar esos factores son: la estabilidad macroeconómica, el esfuerzo de acumulación a altos niveles y de manera sostenida en el tiempo, la inversión en capacidades humanas y la formación de instituciones coherentes y eficientes para la gestión y coordinación. El tránsito hacia una sociedad socialista se ha generado desde las diferentes condiciones de desarrollo de cada región que haya optado por este sistema, de ahí que deben tenerse en cuenta esos elementos distintivos para aplicar un modelo socialista que se adapte a cada sociedad.

\section{Cuba}

Tras el triunfo revolucionario cubano en 1959, el país se encontraba en una difícil situación donde la economía - pequeña y abierta- dependía completamente del imperialismo norteamericano, en los marcos de una profunda deformación estructural donde la agroindustria azucarera era prácticamente el único sector de producción en la Isla. Producía y exportaba un reducido número de productos a los Estados Unidos, mientras dependía de ese mercado para la importación de productos para el abastecimiento de la población. Esta deformación fue heredada desde su condición de colonia española, situación que se acentuó cuando pasó a ser neocolonial de los Estados Unidos.

En los primeros años de la transformación, desde 1959 a 1963, Cuba implementó cambios importantes en pos de atenuar y reconfigurar la situación económica que afrontaba la Isla; 
tomó como medias de industrialización acelerada, la Ley de alquileres, las leyes de Reforma Agraria para diversificar la agricultura del país, entre otras.

A partir del cambio en las relaciones económicas internacionales de $\mathrm{Cuba}$, del incremento de los lazos con la URSS a través de la oferta de abastecer el mercado soviético, el azúcar se convirtió en el sector pivote del desarrollo económico sobre la base de la agroindustria azucarera, por tanto, no era un desarrollo equilibrado. El ingreso de Cuba al Consejo de Ayuda Mutua Económica (CAME) en 1972 fue uno de los motores impulsores que impactó directamente a la economía nacional, el azúcar se consolida como "locomotora" del resto de la economía. Aunque toda esta situación conllevó, durante el período de 1964 a 1975, a que la dinámica economía cubana se hiciera más dependiente aún de un producto primario de exportación. En los 80, a partir del auge neoliberalismo y el surgimiento de la teoría estructuralista de la CEPAL, en América Latina en general y particularmente en Cuba comenzó a darse un proceso de industrialización, sin embargo, tampoco generó muchos resultados positivos debido a las restricciones estructurales y la baja eficiencia del sistema económico cubano. Pero al referirnos a los aspectos sociales, nuestra nación ha alcanzado importantes logros, siendo el primer país de América Latina en incorporar el mejoramiento social a su estrategia de desarrollo como principal prioridad.

La crisis de los años noventa, causada -además de los factores domésticos y estructurales de nuestra economía- por elementos externos y coyunturales, trajo consigo enormes consecuencias para nuestra economía y condujo al país al llamado período especial. A partir de ese momento no se pudo hablar sobre una estrategia de desarrollo para Cuba. La situación heredada por la crisis - aunque en menor medida- perduran aún hasta nuestros días, sin embargo, con los esfuerzos realizados por la Revolución cubana, se han dado importantes acontecimientos positivos que han permitido avanzar hacia la recuperación económica, en especial después del año 2004, por ejemplo con el aprovechamiento de las ventajas competitivas adquiridas mediante la potenciación de los servicios profesionales, que hoy representan el principal rubro de exportación de la Isla, dándose inicio a una nueva etapa de desarrollo económico. A partir de 2011, con el proceso de actualización de modelo económico, las estrategias, en los marcos de la Revolución Socialista, comienzan a tener como base un socialismo próspero y sostenible donde se mantiene la especialización externa, se enfatiza en la necesidad de conducir la economía con mayor eficiencia y lograr una mayor diversificación económica. A partir de esos años (2010-2012) se comienza a tener por primera vez en Cuba una visión de país.

\section{Viet Nam}

Viet Nam es un país que ha sufrido casi un siglo por la guerra. Después del triunfo de la Revolución en 1975, la nación asiática se encontraba en condiciones muy difíciles de desarrollo, donde la guerra había destruido, casi en su totalidad, los recursos con que contaba. Fue un contexto muy difícil para el país, no sólo desde el punto de vista económico, sino que 
también abarcó los aspectos sociales. Prevaleció una escasez extrema en la producción, hasta el punto en que permaneció una situación de inanición desde el período de la guerra hasta finales de los años setenta, donde murieron casi 2 millones de personas. A partir de ese momento, el país comenzó a tomar medidas para la innovación como respuesta a las consecuencias de la guerra y a la necesidad de desarrollarse.

El llamado proceso de innovación “Doi Moi” se inició en 1986 y dio paso de una economía planificada a una economía de mercado. Como resultado de este proceso, Viet Nam logró incrementar el PIB de manera consecutiva desde el año 1986 hasta

1997, con un crecimiento medio anual de 6,6\%. La inflación, que era un número de tres cifras, disminuyó en 12,75\% en 1995 y 4,5\% en 1996. El logro más significativo para Viet Nam fue pasar de un país con elevados niveles de escasez de producción a un país exportador de arroz.

En este período comprendido entre 1986 y 1997 se dieron cambios políticos y económicos. En la agricultura, se eliminó la forma de producción planificada, disminuyó el peso relativo de la cooperativa agropecuaria, se empezó a dar derecho al uso de la tierra a los productores individuales para motivar la producción agrícola, el precio de los productos se comenzó a determinar por el mercado. Con las nuevas políticas aplicadas por el Estado surgieron nuevas leyes, como es el caso de la Ley de inversión extranjera en 1990, la Ley de los cuentapropistas y la Ley empresarial en 1991. Los cambios políticos en este período contribuyeron al incremento de la eficiencia de las empresas, se establecieron las relaciones diplomáticocomerciales y se empezó a impulsar las fuentes externas de financiamiento como la inversión extranjera directa.

De 1997 al 2007, Viet Nam continuó impulsando las medidas necesarias para el proceso de innovación. Uno de los elementos más importantes en este período fueron los cambios que favorecieron a las actividades comerciales de las empresas, estableciendo el mercado de valores, donde se motivó el incrementó de la inversión financiera en ellas. Durante este periodo, Viet Nam también estableció relaciones diplomáticas con más de

150 países y comerciales con más de 190 países y regiones, entrando en las organizaciones comerciales más importantes en el mundo como ASEAN, Organización Mundial del Comercio en 2007 y ha firmado múltiples Tratados de Libre de Comercio con los países asiáticos y los países miembros de la Unión Europa. En estos años tuvo lugar un incremento notable de la economía, pero aún dependía mucho de las inversiones extranjeras y por lo tanto, no era un crecimiento sostenido en el tiempo. Respecto a los aspectos sociales, se incrementó el IDH, también el nivel de longevidad y el nivel escolar de la población. 
Desde 2007 hasta hoy, aunque hay muchos indicadores que se encuentran por debajo del nivel internacional, el país ha tenido, de manera general, un cambio notable. El PIB se ha incrementado 35 veces más respecto al año 1986, desde 6,3 billones de dólares a

224.7 billones. El PIB per cápita, por su parte, aumentó de 80 dólares a 2567, disminuyendo cada vez más la brecha que existe entre el PIB per cápita del país con respecto a los restantes países del mundo. La vida de la población ha mejorado enormemente, donde el ingreso medio por persona se incrementó y hoy día Viet Nam pertenece al grupo de países de renta media.

\section{Desarrollo y crecimiento. Una comparación}

Resulta interesante comparar cómo ha incidido el crecimiento alcanzado -en su período de tránsito al Socialismo- en el desarrollo en las economías vietnamita y cubana. Como indicador del desarrollo se tomará el Índice de Desarrollo Humano (IDH) que, aunque tiene sus limitantes, no dando cuenta del nivel de desarrollo alcanzado por una política pública en particular ni del poder adquisitivo de la población, es el indicador más conocido y aceptado hasta la actualidad para medir en este sentido, ya que es multidimensional, abarcando la longevidad, la educación y los ingresos para dar cuenta del impacto en el fortalecimiento y ampliación de las capacidades personales.

Esta comparación se realizará mediante un análisis econométrico, midiendo el impacto que ha tenido el PIB per cápita en el IDH para ambas naciones desde el año 1995 hasta el 2016. Se tomará el indicador PIB como medida de crecimiento, el cual es el más aceptado a nivel internacional, aunque resulta necesario destacar que igualmente posee limitaciones, ya que hay determinados aspectos que no se pueden medir como: el sobreajuste por inflación, la producción en el hogar, la actividad económica subterránea, la calidad del medio ambiente, entre otros. Además, para el caso específico de Cuba, su medición presenta diversas dificultades, por lo que los valores registrados no son exactos.

Es importante señalar también que el análisis se realizará en términos per cápita para que el indicador sea comparable para ambos países; y que las series serán analizadas en términos logarítmicos para que los datos se acerquen más a la realidad económica.

El análisis arrojó como resultado que en Viet Nam, mientras que el PIB per cápita aumenta en un punto porcentual, el IDH decrece 1 en 0,04\% (tabla No.1); mientras que, para Cuba, ese aumento en $1 \%$ en el PIB, provoca un incremento en el IDH en un 0,14\% (tabla No.2). 
ISSN: 2600-5859

Tabla 1. Ecuación estimada del IDH sobre el PIB sobre el PIB per cápita de Viet Nam (1995-2016)

\begin{tabular}{|c|c|c|c|c|}
\hline \multicolumn{5}{|c|}{$\begin{array}{l}\text { Dependent Variable: LPIB } \\
\text { Method: Least Squares } \\
\text { Date: } 12 / 09 / 18 \text { Time: } 09: 18 \\
\text { Sample: } 19952016 \\
\text { Included observations: } 22\end{array}$} \\
\hline Variable & Coefficient & Std. Error & t-Statistic & Prob. \\
\hline $\begin{array}{l}\text { LSALUD } \\
\text { C } \\
\text { @TREND }\end{array}$ & $\begin{array}{l}0.324384 \\
7.123779 \\
0.050788\end{array}$ & $\begin{array}{l}0.160448 \\
0.262070 \\
0.007475\end{array}$ & $\begin{array}{l}2.021733 \\
27.18270 \\
6.794654\end{array}$ & $\begin{array}{l}0.0575 \\
0.0000 \\
0.0000\end{array}$ \\
\hline $\begin{array}{l}\text { R-squared } \\
\text { Adjusted R-squared } \\
\text { S.E. of regression } \\
\text { Sum squared resid } \\
\text { Log likelihood } \\
\text { F-statistic } \\
\text { Prob(F-statistic) }\end{array}$ & $\begin{array}{l}0.961399 \\
0.957336 \\
0.088812 \\
0.149863 \\
23.66321 \\
236.6084 \\
0.000000\end{array}$ & \multicolumn{2}{|c|}{$\begin{array}{l}\text { Mean dependent var } \\
\text { S.D. dependent var } \\
\text { Akaike info criterion } \\
\text { Schwarz criterion } \\
\text { Hannan-Quinn criter. } \\
\text { Durbin-Watson stat }\end{array}$} & $\begin{array}{r}8.327171 \\
0.429971 \\
-1.878473 \\
-1.729695 \\
-1.843426 \\
0.875603\end{array}$ \\
\hline
\end{tabular}

Fuente: Elaboración propia a partir del paquete econométrico Eviews

Tabla 2. Ecuación estimada del IDH per cápita de Cuba (1995-2016)

\begin{tabular}{|c|c|c|c|c|}
\hline \multicolumn{5}{|c|}{$\begin{array}{l}\text { Dependent Variable: LPIB } \\
\text { Method: Least Squares } \\
\text { Date: } 12 / 09 / 18 \text { Time: } 07: 50 \\
\text { Sample (adjusted): } 19952015 \\
\text { Included observations: } 21 \text { after adjustments }\end{array}$} \\
\hline Variable & Coefficient & Std. Error & t-Statistic & Prob. \\
\hline $\begin{array}{l}\text { LSALUD } \\
\text { C } \\
\text { @TREND }\end{array}$ & $\begin{array}{l}0.861730 \\
4.170137 \\
0.100253\end{array}$ & $\begin{array}{l}0.216538 \\
0.330433 \\
0.004249\end{array}$ & $\begin{array}{l}3.979582 \\
12.62024 \\
23.59270\end{array}$ & $\begin{array}{l}0.0009 \\
0.0000 \\
0.0000\end{array}$ \\
\hline $\begin{array}{l}\text { R-squared } \\
\text { Adjusted R-squared } \\
\text { S.E. of regression } \\
\text { Sum squared resid } \\
\text { Log likelihood } \\
\text { F-statistic } \\
\text { Prob(F-statistic) }\end{array}$ & $\begin{array}{l}0.985357 \\
0.983730 \\
0.089320 \\
0.143605 \\
22.54698 \\
605.6370 \\
0.000000\end{array}$ & \multicolumn{2}{|c|}{$\begin{array}{l}\text { Mean dependent var } \\
\text { S.D. dependent var } \\
\text { Akaike info criterion } \\
\text { Schwarz criterion } \\
\text { Hannan-Quinn criter. } \\
\text { Durbin-Watson stat }\end{array}$} & $\begin{array}{r}6.589494 \\
0.700259 \\
-1.861617 \\
-1.712400 \\
-1.829233 \\
1.483885\end{array}$ \\
\hline
\end{tabular}

Fuente: Elaboración propia a partir del paquete econométrico Eviews

El impacto del crecimiento en el desarrollo en Cuba es superior al de Viet Nam, lo cual podría estar indicando que el socialismo en la experiencia cubana ha influido de un modo más significativo en el desarrollo del país, sin embargo, este resultado se debe, esencialmente, a que Cuba se enfoca más en aspectos sociales que Viet Nam, por ejemplo, dedica anualmente una mayor proporción del presupuesto a la esfera de la salud. Al analizar el impacto de los gastos anuales en salud sobre el PIB, se puede deducir, tal como se esperaba, que en Cuba tiene un mayor impacto que en la economía asiática; para Viet Nam, un aumento en un $1 \%$ 
en los gastos en salud representa un incremento de un $0,34 \%$ en el crecimiento económico (tabla No.3), mientras que para Cuba ese impacto es del 86\% (tabla No.4).

Tabla N 3. Ecuación estimada sobre los gastos de salud de Viet Nam (1995-2016)

\begin{tabular}{|c|c|c|c|c|}
\hline \multicolumn{5}{|c|}{$\begin{array}{l}\text { Dependent Variable: LPIB } \\
\text { Method: Least Squares } \\
\text { Date: 12/09/18 Time: 09:18 } \\
\text { Sample: } 19952016 \\
\text { Included observations: } 22\end{array}$} \\
\hline Variable & Coefficient & Std. Error & t-Statistic & Prob. \\
\hline $\begin{array}{l}\text { LSALUD } \\
\quad \mathrm{C} \\
\text { @TREND }\end{array}$ & $\begin{array}{l}0.324384 \\
7.123779 \\
0.050788\end{array}$ & $\begin{array}{l}0.160448 \\
0.262070 \\
0.007475\end{array}$ & $\begin{array}{l}2.021733 \\
27.18270 \\
6.794654\end{array}$ & $\begin{array}{l}0.0575 \\
0.0000 \\
0.0000\end{array}$ \\
\hline $\begin{array}{l}\text { R-squared } \\
\text { Adjusted R-squared } \\
\text { S.E. of regression } \\
\text { Sum squared resid } \\
\text { Log likelihood } \\
\text { F-statistic } \\
\text { Prob(F-statistic) }\end{array}$ & $\begin{array}{l}0.961399 \\
0.957336 \\
0.088812 \\
0.149863 \\
23.66321 \\
236.6084 \\
0.000000\end{array}$ & \multicolumn{2}{|c|}{$\begin{array}{l}\text { Mean dependent var } \\
\text { S.D. dependent var } \\
\text { Akaike info criterion } \\
\text { Schwarz criterion } \\
\text { Hannan-Quinn criter. } \\
\text { Durbin-Watson stat }\end{array}$} & $\begin{array}{r}8.327171 \\
0.429971 \\
-1.878473 \\
-1.729695 \\
-1.843426 \\
0.875603\end{array}$ \\
\hline
\end{tabular}

Fuente: Elaboración propia a partir del paquete econométrico Eviews

Tabla N 4. Ecuación estimada sobre los gastos de salud de Cuba (1995-2016)

\begin{tabular}{|c|c|c|c|c|}
\hline \multicolumn{5}{|c|}{$\begin{array}{l}\text { Dependent Variable: LPIB } \\
\text { Method: Least Squares } \\
\text { Date: 12/09/18 Time: } 07: 50 \\
\text { Sample (adjusted): } 19952015 \\
\text { Included observations: } 21 \text { after adjustments }\end{array}$} \\
\hline Variable & Coefficient & Std. Error & t-Statistic & Prob. \\
\hline $\begin{array}{l}\text { LSALUD } \\
\text { C } \\
\text { @TREND }\end{array}$ & $\begin{array}{l}0.861730 \\
4.170137 \\
0.100253\end{array}$ & $\begin{array}{l}0.216538 \\
0.330433 \\
0.004249\end{array}$ & $\begin{array}{l}3.979582 \\
12.62024 \\
23.59270\end{array}$ & $\begin{array}{l}0.0009 \\
0.0000 \\
0.0000\end{array}$ \\
\hline $\begin{array}{l}\text { R-squared } \\
\text { Adjusted R-squared } \\
\text { S.E. of regression } \\
\text { Sum squared resid } \\
\text { Log likelihood } \\
\text { F-statistic } \\
\text { Prob(F-statistic) }\end{array}$ & $\begin{array}{l}0.985357 \\
0.983730 \\
0.089320 \\
0.143605 \\
22.54698 \\
605.6370 \\
0.000000\end{array}$ & \multicolumn{2}{|c|}{$\begin{array}{l}\text { Mean dependent var } \\
\text { S.D. dependent var } \\
\text { Akaike info criterion } \\
\text { Schwarz criterion } \\
\text { Hannan-Quinn criter. } \\
\text { Durbin-Watson stat }\end{array}$} & $\begin{array}{r}6.589494 \\
0.700259 \\
-1.861617 \\
-1.712400 \\
-1.829233 \\
1.483885\end{array}$ \\
\hline
\end{tabular}

Fuente: Elaboración propia a partir del paquete econométrico Eviews

Cuba se ha caracterizado históricamente, después del triunfo de la Revolución, por centrar su política en obtener logros sociales, los cuales han ido en ascenso con el paso de los años. La actualidad no constituye una excepción en este sentido, puesto que fiel a la concepción socialista que sitúa al ser humano en el centro de la práctica política y la gestión gubernamental, aprobado por Asamblea Nacional en la Ley del Presupuesto para 2017, se destinó el $72 \%$ de los gastos corrientes del Estado a los servicios sociales básicos vinculados a la calidad de vida de la población y las prestaciones de la seguridad social. A la Salud Pública y la Asistencia Social se destinaron 10 mil 206 millones 200 mil pesos, que significa el $28 \%$ del gasto corriente y representa el $11 \%$ del PIB. Estas cifras permitieron sostener los 
servicios en 12400 unidades asistenciales, entre ellas, 151 hospitales, 451 policlínicos, 10 782 consultorios de médicos de la familia, 110 clínicas estomatológicas, 147 hogares de ancianos, 265 casas de abuelos y 30 hogares de impedidos físicos; y se respaldó la prestación de servicios de salud para cifras de 1 millón 120 mil ingresos hospitalarios en más de 45 mil camas, 86 millones de consultas médicas y unas 28 millones 500 mil consultas estomatológicas.

Sin embargo, ni lo plantea ahora, ni el hecho de que Cuba historialmente posea un IDH superior al de Viet Nam (grafico No.1), está implicando que sea un país más desarrollado que la nación asiática.

Figura N 1. IDH de Cuba y Viet Nam (1995-2016)

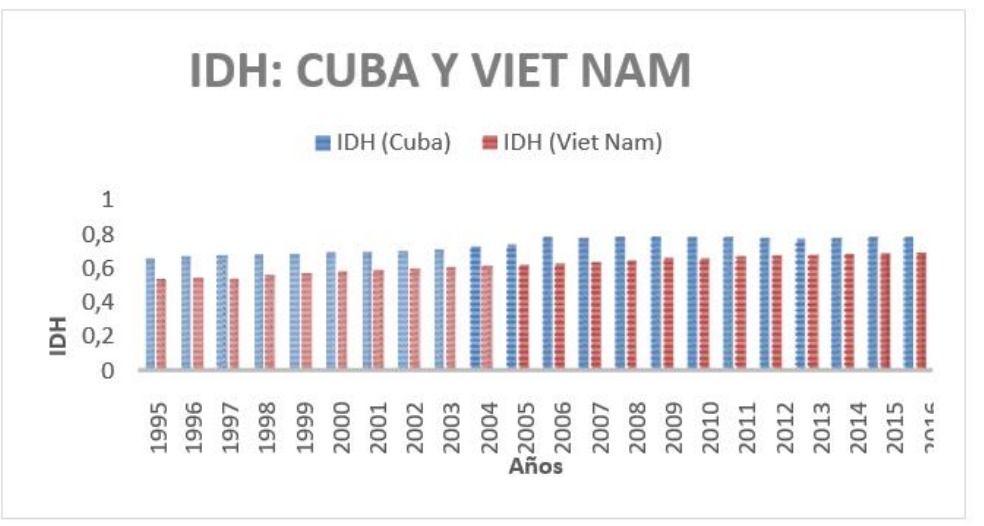

Fuente: Elaboración propia a partir del paquete econométrico Eviews

Este planteamiento se debe a que Viet Nam, en su condición de Socialismo de Mercado, centra más sus políticas en los aspectos económicos, destina sus gastos mayormente a la inversión productiva, aquella que generará rentabilidad en el mediano o largo plazo; a diferencia de Cuba, que sus inversiones se dirigen en función de la sociedad. Por ejemplo, Viet Nam ha mostrado importantes niveles de inversión extranjera directa (IED), la cual ha demostrado su importancia para el proceso de desarrollo económico del país. Desde su condición inicial de desarrollo bajo, las fuentes externas de financiamiento constituyeron un importante eslabón para equilibrar la balanza de pagos y alcanzar mayores niveles de desarrollo económico y social, destacando que los resultados positivos que han obtenido en el largo plazo, no hubieran sido posible sin la intervención estatal2, rasgo que caracteriza al Socialismo; el Gobierno, durante el período de tránsito, ha tenido en cuenta los efectos negativos resultantes de errores en las políticas para mejorar el camino de construcción de una economía fortalecida. De modo que su crecimiento ha tenido una tendencia notablemente creciente y potencialmente mayor -en términos de velocidad de crecimiento- al experimentado por la economía cubana en los últimos años, cuya tasa de crecimiento ha ido en ascenso pero es, en términos medios, cada vez menor. 


\section{Conclusiones}

- En No se puede hacer referencia al éxito o fracaso total de las experiencias socialistas, tampoco existe un modelo general a seguir en el tránsito al Socialismo, las pautas a seguir quedarán determinadas por las condiciones en que se desarrolle cada nación. Cuba y Viet Nam son ejemplos históricos de países que afrontaron una larga historia de guerra y son subdesarrollados, sin embargo, Viet Nam planteó desarrollarse con una economía de mercado mientras que Cuba avanza en su camino desde los principios después de una economía planificada. Ningún modelo socialista hasta ahora implementado en cada país ha sido perfecto, pero sí se pueden sentar las bases para la obtención de mejores resultados en el camino hacia la sociedad socialista, por lo tanto, se trata de un proceso de adaptación a la realidad histórico-concreta de cada territorio, teniendo en consideración los aspectos positivos y negativos de la implementación ejecutada, máxime cuando se tiene en cuenta que el mundo se mueve en un contexto cada vez más globalizado.

- Resulta importante entender que -como se mencionó con anterioridad- el Socialismo genera desarrollo, de hecho, tiene como meta alcanzar un pleno desarrollo humano; pero también el desarrollo es condición necesaria para alcanzar el Socialismo, ya que ese desarrollo humano se logra sobre la base de un crecimiento económico. Una sociedad socialista requiere de una economía desarrollada hasta el punto en que tras el alcance de la sociedad donde se produzca en función del beneficio social, se logre producir según las necesidades, se producirán valores de uso que serán distribuidos a cada individuo con el objetivo de satisfacer cada una de sus necesidades; esto requerirá un nivel elevado de desarrollo de las fuerzas productivas. El salario será sustituido por un acceso libre a todos los bienes que necesiten los productores para satisfacer sus necesidades como retribución al trabajo realizado; lo cual requiere de un nivel superior de la consciencia social que sólo será posible con una presencia abundante de bienes en la sociedad. Además, para erradicar la desigualdad económica y social -requerido por el Socialismo- es imprescindible, entre otros aspectos, la eliminación real de la desigualdad de género humano, elemento que se incluye dentro del concepto de desarrollo.

\section{Referencias bibliográficas.}

Arruda de Sampaio, Plinio. Mundialización del capital y crisis del desarrollo nacional [CLASE]. En: Curso virtual "Actualidad del pensamiento crítico latinoamericano sobre la problemática del desarrollo nacional." (Programa Latinoamericano de 
Educación a Distancia, Centro Cultural de la Cooperación, Buenos Aires, Abril de 2008).

Chang, Ha-Joon. "Patada a la escalera: La verdadera historia del libre comercio", 2003

CEPAL: Estudio Económico de América Latina y el Caribe. Cuba, 2017

Datos publicados por la Oficina Nacional de Estadística e Información (ONEI)

López Pardo, Cándido. "Desarrollo humano en América Latina y el Caribe: eficacia y eficiencia", 2002

Pérez Soto, Olga. "El socialismo como ciencia económica del desarrollo. Lo necesario y posible". Editorial Universitaria Félix Varela, 2018 (Folleto 4)

Pérez Soto, Olga. "La ciencia económica de la transición socialista. La cuarta identidad". Editorial Universitaria Félix Varela, 2018 (Folleto 7)

PNUD: Informe sobre desarrollo humano. Ediciones Mundi-Prensa

Sitio web es.scibd.com 
Para citar el artículo indexado.

Rodríguez Piñeiro, C., \& Dang Do Loc, A. P. (2019). El proceso de desarrollo en las experiencias de construcción socialista de Viet Nam y Cuba: un análisis comparado. ConcienciaDigital, 2(2), 41-52. https://doi.org/10.33262/concienciadigital.v2i2.943

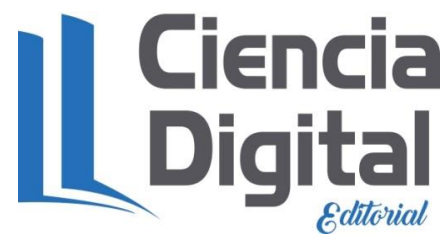

El artículo que se publica es de exclusiva responsabilidad de los autores y no necesariamente reflejan el pensamiento de la Revista Conciencia Digital.

El articulo queda en propiedad de la revista y, por tanto, su publicación parcial y/o total en otro medio tiene que ser autorizado por el director de la Revista Conciencia Digital.

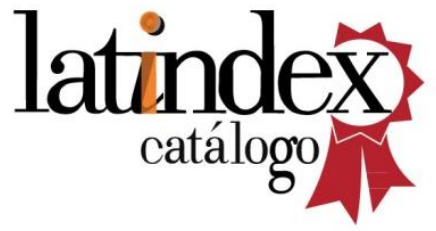

\section{Conciencia}

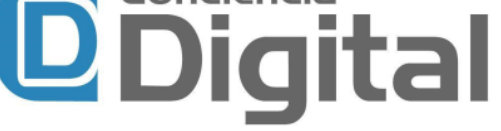

